\title{
O trabalho em rede nos casos de alta complexidade e de alta vulnerabilidade: a experiência de uma UBS
}

\author{
Health care networking in cases of high \\ complexity and high vulnerability: \\ the experience of an Health Center
}

\section{El trabajo en redes en los casos de alta complejidad y alta vulnerabilidad: la experiencia de una Unidad Básica de Salud}

\author{
Carmen Ligia Cucê Nobre* \\ Adriano de Oliveira** \\ Julia Hatakeyama Joia*** \\ Luiza Santa Cruz* \\ Luiza Franco* \\ Keyla Raquel F. de Oliveira**** \\ Janaina Eleuterio**** \\ Maria Luiza Rezende**** \\ Silvia Regina Rocha****
}

*Universidade de São Paulo, Ribeirão Preto, SP, Brasil.

**Universidade Estadual Paulista, UNESP, Bauru, SP, Brasil.

***Pontifícia Universidade Católica de São Paulo, PUC-SP, São Paulo, SP, Brasil.

****Secretaria Municipal de Saúde, São Paulo, SP, Brasil.

Contribuição dos autores:

CLCN: coleta e sistematização de dados e elaboração e revisão do manuscrito.

AO: realização de oficinas de escrita e elaboração e revisão do manuscrito.

JHJ: realização de oficinas de escrita e elaboração e revisão do manuscrito.

LSC: coleta e sistematização de dados e elaboração e revisão do manuscrito.

LF: coleta e sistematização de dados e elaboração e revisão do manuscrito.

KRFO: coleta e sistematização de dados e elaboração e revisão do manuscrito.

JE: coleta e sistematização de dados e elaboração e revisão do manuscrito.

MLR: coleta e sistematização de dados e elaboração e revisão do manuscrito.

SRR: coleta e sistematização de dados e elaboração e revisão do manuscrito.

E-mail para correspondência: Julia Hatakeyama Joia - julia.joia@gmail.com

Recebido: 02/12/2016

Aprovado: 01/04/2017 


\section{Resumo}

Este estudo tem como objetivo apresentar e analisar os processos de formação de redes de atenção à saúde em torno de casos complexos e em contextos de alta vulnerabilidade, atendidos pela Estratégia Saúde da Família na UBS XXX, na região da Brasilândia, São Paulo. Tais processos foram desenvolvidos a partir de oficinas de escrita realizadas pelo Pró-Saúde-PUCSP em parceria com a Supervisão Técnica de Saúde da Freguesia do Ó/Brasilândia da Secretaria Municipal de Saúde de São Paulo. As oficinas se constituíram como forma de mobilizar o relato de experiências e a reflexão sobre a prática profissional. Partiu-se de três casos índices atendidos na Unidade Básica de Saúde que demandaram uma configuração de redes ampliadas de cuidado que se estenderam para além do atendimento multiprofissional da Unidade e do apoio do Núcleo de Apoio à Saúde da Família (NASF). Foram tecidas reflexões em torno dos diferentes modelos de assistência em saúde, da resolubilidade de tais casos e a da corresponsabilidade dos atores envolvidos no cuidado. Concluiu-se que a formação de rede aproxima os profissionais do território, explora a potencialidade de assistência e possibilita uma abordagem mais integrada, proporcionando um atendimento mais humanizado.

Palavras-chave: Estratégia Saúde da Família; Atenção Primária à Saúde; Centros de Saúde, Vulnerabilidade em Saúde.

\section{Abstract}

This study aims to present and analyze the health care network formation processes around cases of high complexity and high vulnerability served by the Family Health Strategy at UBS XXXX, in the region of Brasilândia, in São Paulo, Brazil. Such processes have been developed from writing workshops conducted by PUC-SP Pro-Health in partnership with Health Supervision Technical FÓ/Brasilândia SMS/ SP. The workshops were formed as a way to mobilize the narrative of experiences and reflection about the professional practices. The work was started from three index cases treated at UBS, which required a configuration of extended care networks that extended beyond the multidisciplinary care of the Unit and the support of NASF. Reflections were woven around the different health care models, of the possibility of resolving such cases and the responsibility of the actors involved in the care. It was concluded that the network formation approximates professionals of the territory, explores the assistance capability and makes it possible a more integrated approach, providing a more humanized care.

Keywords: Family Health Strategy; Primary Health Care; Health Centers, Health Vulnerability.

\section{Resumen}

Este estudio tiene como objetivo presentar y analizar los procesos de formación de la red de atención de salud en torno de casos complejos y en contextos de alta vulnerabilidad, atendidos por la Estrategia Salud de la Familiar en la Unidad Básica de Salud (UBS) XXXX, en la región de Brasilândia, en Sao Paulo, Brasil. Tales procesos se han desarrollado a partir de talleres de escritura realizadas por ProSalud-PUC-SP en colaboración con la Supervisión Técnica de Salud de la "Freguesia do Ó"/ Brasilândia Secretaria Municipal de São Paulo. Los talleres se formaron como una forma de movilizar la narración de experiencias y la reflexión sobre las prácticas profesionales. El trabajo se inició a partir de tres casos índice tratados en la UBS, que requerían una configuración de redes ampliadas de atención que se extendieron más allá de la atención multidisciplinaria de la Unidad y del apoyo del Núcleo de Apoyo a la Salud (NASF). Reflexiones fueron tejidas en torno a los diferentes modelos de atención de la salud, a la posibilidad de resolver este tipo de casos y a la corresponsabilidad de los actores implicados en la atención. Se concluyó que la formación de la red aproxima a los profesionales del territorio, explora la capacidad de asistencia y hace posible un enfoque más integrado, proporcionando una atención más humanizada.

Palabras claves: Estrategia de Salud Familiar; Atención Primaria de Salud; Centros de Salud, Vulnerabilidad en Salud. 


\section{Introdução}

As reflexões apresentadas neste texto foram disparadas por meio de oficinas de escrita realizadas em uma UBS, promovidas pelo Pró-Saúde/ PUC-SP, o Programa Nacional de Reorientação da Formação Profissional em Saúde do Ministério da Saúde, fruto da parceria da PUC-SP com a Supervisão Técnica de Saúde da Fó-Brasilândia, no distrito sanitário da Coordenadoria de Saúde Norte/ Secretaria Municipal de Saúde de São Paulo ${ }^{1}$. No seu desenvolvimento, partiu-se da premissa inicial do Pró-Saúde de que os profissionais dos serviços seriam referência para intervenções que envolvessem a assistência ou a produção de procedimentos técnicos-operativos, mas também seriam parceiros de docentes e estudantes na produção de referências teóricas/conhecimento de formação em saúde. As oficinas constituíram-se em espaços de reflexão e produção de conhecimento a partir da problematização e registro de experiências nos processos de trabalho de profissionais dessa Unidade de Saúde, configurando um modo de aproximação entre a universidade e os serviços de saúde.

Como estratégia de sistematização e registro das experiências dos trabalhadores da rede de saúde da FÓ/Brasilândia, foram realizados 06 encontros com profissionais da Estratégia Saúde da Família (ESF) da Unidade e do Núcleo de Apoio da Saúde da Família (NASF) de referência das UBSs XXX, YYY e ZZZ. As oficinas foram apoiadas por dois tutores, que tinham por função promover e facilitar a discussão e a produção de textos que expressassem o conhecimento gerado na pratica cotidiana do serviço de saúde. A forma de trabalho, que foi denominada Oficina de Escrita, privilegiou a constituição de um espaço no qual os trabalhadores eram incentivados a recordar experiências que consideravam significativas em suas trajetórias naquela UBS e que retratavam o processo de construção de um trabalho articulado ao Modelo Assistencial de Atenção Integral à Saúde do Sistema Único de Saúde. Nos primeiros encontros, o grupo pôde elencar e produzir reflexões coletivas sobre as experiências consideradas mais desafiantes enfrentadas pelas equipes. Os profissionais envolvidos puderam estabelecer alguns itinerários, buscando analisar também a maneira como produzem o trabalho em redes e seus impactos no cuidado em saúde dos casos discutidos. Após este momento inicial de aquecimento, o grupo foi orientado a eleger um eixo temático que pudesse nortear a produção coletiva de um texto sobre suas práticas. $\mathrm{O}$ eixo eleito foi "A formação de redes nos casos de alta vulnerabilidade e complexidade", e, nos encontros seguintes, as equipes puderam debruçar-se sobre casos complexos para extrair destes alguns norteadores comuns de trabalho e desdobrar reflexões sobre impasses e desafios. A tarefa para os integrantes das equipes era metabolizar experiências vividas, a fim de construir um discurso coletivo sobre seu fazer e suas práticas e compartilhá-las com o campo da produção de conhecimento em saúde. Os tutores, neste processo, gravaram as discussões dos encontros em aúdio, fazendo transcrições que orientaram a escrita dos participantes, e também atuaram como organizadores das diversas "idas e vindas" do texto, que, mesmo após a finalização das oficinas, foi sendo construído e revisado pelo conjunto de autores.

\section{Estratégia Saúde da Família: implicações sobre os modos de fazer o cuidado}

O modelo de assistência em saúde vem se transformando ao longo dos anos. Com a implantação do SUS, em 1990, foram elaborados princípios de atendimento ao usuário que visassem garantir a universalidade, integralidade e equidade da atenção em saúde. Como desdobramento do PACS (Programa de Agentes Comunitários de Saúde), formulado no início da década de $1990^{2}$, o então denominado Programa Saúde da Família (PSF) ${ }^{3}$, concebido em 1994, representou mais um passo na garantia do atendimento integral, ao redirecionar a atenção em saúde dos usuários no contexto de seu território de vida, dando escuta aos seus anseios e necessidades, incorporando ações nos campos de prevenção, promoção, recuperação e reabilitação de doenças e agravos à saúde. Em 2006 passou a ser reconhecido como Estratégia da Saúde da Família, na tentativa de ressaltar sua função estratégica e central na política de saúde em detrimento da ideia de se tratar de um programa específico e delimitado, uma vez que trabalha na perspectiva da superação do modelo de assistência curativista e hospitalocêntrico, centrado somente na consulta especializada ${ }^{2}$.

Tais pressupostos de trabalho vêm promovendo transformações importantes na Atenção Básica ( $\mathrm{AB})$, reorientando o modelo assistencial nas Unidades Básicas de Saúde (UBSs). Essa nova organização da atenção em saúde propicia o maior 
contato do sistema de saúde com a vida das pessoas, bem como facilita o reconhecimento do território e das demandas em saúde existentes em uma coletividade, na própria comunidade. Além disso, favorece a formação de vínculos entre profissionais de saúde e população assistida e cria possibilidades outras de ações na promoção da saúde da população.

No cenário da incorporação da ESF, toma centralidade a figura do Agente Comunitário de Saúde (ACS), cuja presença extrapola o espaço físico reservado do serviço de saúde, na direção de se aproximar dos contextos existenciais dos usuários. Além de ser um membro integrante do território, conhecedor da realidade vivida pela população, enriquece o nível de informações sobre a saúde da comunidade adstrita, ao sair do equipamento de saúde e ir até o usuário, aproximando-se de seu modo de viver.

$\mathrm{Se}$, anteriormente, o modelo de atenção era organizado nos moldes de ambiente hospitalar ou sala de consulta, fundamentado em saberes específicos, pontuados na figura dos especialistas (profissionais únicos, médico, psicólogo, enfermeiro), a inovação da ESF visa ampliar os olhares e superar a fragmentação dos saberes. $\mathrm{O}$ trabalho realizado isoladamente pautado em diferentes áreas do saber, a clínica baseada na doença, o paradigma de "queixa-conduta", a lógica da segmentação gerando a prática de encaminhamento para diversos profissionais são ranços de um modelo de atenção em saúde que não abarca a complexidade dos sujeitos e das comunidades.

Com a implantação da ESF, foi possível uma maior aproximação dos profissionais da saúde aos diferentes registros da experiência do sujeito em sua multiplicidade, produzindo um campo favorável à efetivação da integralidade da atenção à saúde. Os atendimentos pontuais em saúde como resposta única, assim como suas práticas protocolares e burocratizadas, foram rechaçados na busca de outras formas de se compreender os processos de saúde-doença. Ao se deparar com a singularidade do sujeito, ao ouvir seus relatos de como vivencia o momento de seu adoecimento, inserido no contexto de sua história de vida, novos horizontes vão se desvelando.

Como parte de um processo de fortalecimento da ESF, em 2008 foi criado o Núcleo de Apoio à Saúde da Família (NASF), que passou a incorporar o grupo de trabalhadores da atenção primária à saúde ${ }^{4}$. O NASF foi criado com a finalidade de potencializar e horizontalizar o diálogo e a articulação entre as diferentes áreas de saber, propondo uma prática reorganizadora dos cuidados primários de saúde. Além disso, tem o objetivo de contribuir, também, com a ampliação do leque de ações em situações de maior enredamento, visando estratégias mais eficazes diante de processos de saúde-doença complexos.

A composição deste modelo de assistência, no entanto, não está dada de antemão; é algo que vem se construindo segundo certos movimentos e na medida em que determinadas demandas vão se produzindo. A configuração de uma rede de cuidados é permeada de ires-e-vires e requer muitos momentos de discussão e reflexão, podendo gerar controvérsias e diferentes formas de conflito. Portanto, trata-se de um trabalho que exige uma abertura para o imprevisível, pois é constituído e construído na processualidade dos acontecimentos.

\section{Apresentando os casos de alta vulnerabilidade e de alta complexidade da UBS}

No procedimento cotidiano de atendimento à população, muitos caminhos são percorridos. $\mathrm{Na}$ experiência da UBS XXX, localizada no distrito sanitário da Brasilândia, os casos considerados de alta vulnerabilidade e de alta complexidade se apresentam como um desafio cotidiano para a rede de saúde local. A Unidade atende a uma população de mais de 6.400 famílias, perfazendo cerca de 24.000 habitantes. É uma das regiões mais carentes da cidade, com um dos maiores índices de vulnerabilidade social (grupo 6 do Índice Paulista de Vulnerabilidade Social, que circunscreve "vulnerabilidade muito alta"), baixa condição socioeconômica, população bem jovem (cerca de $1 / 3$ entre 0 a 19 anos; $1 / 3$ entre 20 a 39 anos), com alto índice de evasão escolar ${ }^{5}$.

As famílias são constituídas por chefe de família jovem, baixa renda, pouca escolaridade, expressiva quantidade de crianças pequenas, significativo número de gestantes adolescentes, insuficiente apoio familiar. Tudo isso, somado a maior exposição à violência urbana, conforma uma população sujeita a riscos, não só sob o ponto de vista da dificuldade de sair da zona da pobreza, mas também traz complicações aos cuidados e à manutenção do estado de saúde.

Os casos complexos que vivem em condições consideradas de alta vulnerabilidade atendidos 
pela UBS XXX envolvem situações como: maus-tratos à pessoa com deficiência física, dificuldades sócio-econômicas, relacionamentos familiares conturbados, uso de substâncias psicoativas, gestação na adolescência, falta de acessibilidade aos pacientes com graves limitações físico-motoras, jovens com dificuldades para frequentar escola, higienização insuficiente de familiar dependente, moradia em condição precária. A realidade destes usuários suscita demandas recorrentes à UBS, colocando diversas questões: como cuidar da saúde da pessoa quando a realidade na qual está inserida é envolta em outras dimensões sociais que interferem diretamente em seus processos de adoecimento? Como atuar em saúde coletiva num território de alta vulnerabilidade e de violência?

Diante da complexidade de cada caso que chega à UBS, os profissionais da área de saúde são mobilizados a expandirem suas práticas profissionais para além de suas formações e atuações individualizadas. Nestes casos, além da busca de subsídios em outras áreas de saber, e de outras formações profissionais, faz-se necessário também o acionamento de redes ampliadas constituídas de outros serviços da área de saúde e até mesmo de outros setores públicos.

Desse modo, o presente trabalho se debruçou sobre a experiência da ESF nesta UBS e as questões advindas da prática cotidiana de produção de redes de cuidado. A fim de contemplar o objetivo desta comunicação partiu-se do estudo de três casos de alta complexidade e de alta vulnerabilidade que envolveram não só o serviço de saúde da UBS, como também os demais níveis dos serviços de saúde, além de outros setores públicos.

\section{Discussão acerca dos desafios na formação de redes de cuidado para casos complexos e situação de alta vulnerabilidade}

No cotidiano de trabalho da UBS, os casos de alta vulnerabilidade são aqueles que requerem a ampliação dos dispositivos de cuidado, espaços de discussão de caso, exigindo a circulação das suas demandas entre as equipes e também entre outros serviços.

A identificação destes casos é possibilitada, em primeiro lugar, pela aproximação dos ACSs com as famílias do seu território de referência. Em seguida, as situações encontradas e/ou solicitadas são compartilhadas e discutidas em equipe Saúde da Família. Nesse processo, busca-se incluir o usuário, sua família e demais pessoas da comunidade que possam ajudar na elaboração das estratégias de cuidado e apoiar as ações a serem realizadas. Por fim, elabora-se um PTS (Projeto Terapêutico Singular), em que se pode incluir o NASF e outros serviços (da rede de saúde e de outros setores, como Assistência Social, Educação, Habitação, etc), quando necessário.

Dessa forma, pode-se destacar pelo menos cinco níveis de articulação de rede: 1) entre profissionais de uma equipe ampliada (equipe SF/NASF); 2) entre equipes distintas de um mesmo serviço; 3 ) entre diferentes serviços de Saúde (Atenção Básica e Níveis Secundário e Terciário de Atenção em Saúde); 4) entre serviços e equipamentos de outras políticas (Assistência Social, Educação, Habitação, Conselho Tutelar, Ministério Público, etc); 5) entre as equipes de cuidado, a família e a comunidade.

A seguir serão apresentados, brevemente, os três casos considerados emblemáticos, dada a complexidade das suas demandas e os múltiplos desdobramentos que o acompanhamento proporcionou, e a maneira como se articularam as redes frente aos desafios enfrentados pelas equipes SF/NASF.

O primeiro caso é de um paciente jovem que morava sozinho e que permaneceu muitos meses em internação hospitalar, paraplégico, acamado, com úlceras de pressão e infecções de repetição. Foi necessário buscar uma rede ampliada de cuidados que envolvessem a família e vizinhos para os cuidados diários: banho, troca, alimento; o CRAS/ CREAS, na busca de Benefício do INSS e direitos do DPVAT; referência hospitalar para os cuidados clínicos; e, principalmente, a disposição da própria UBS/NASF para os cuidados diários de curativos que duravam cerca de uma hora, incluindo sábado, domingo e feriado, bem como para o apoio emocional ao paciente e seus familiares. Este paciente veio a óbito, causando forte comoção nos envolvidos.

O segundo caso é de uma família numerosa com históricos de doença mental, uso de substâncias psicoativas, gravidez na adolescência, crianças e jovens fora da escola, moradia precária e higienização descuidada. Neste caso, a rede se configurou ao buscar reforços com o CRAS/ CREAS, Conselho Tutelar, CAPS Infantojuvenil, CAPS Álcool e Drogas, escola da região. Toda essa intersetorialidade tinha como objetivo a tentativa de cooperação, numa possível reorganização familiar, onde os direitos humanos pudessem ser preser- 
vados e colocados em prática pelas instituições responsáveis e pela própria família. Atualmente as propostas tiradas em conjunto, inclusive com o grupo familiar, estão em andamento, algumas com maior sucesso, outras em fase de reconstrução e novas pactuações.

O terceiro caso refere-se a um homem com déficit cognitivo, físico e motor que vive literalmente no chão da casa de seus familiares. Sobrevive nu, come e bebe em vasilhas colocadas no chão, locomove-se se arrastando. Metaforicamente é uma sombra familiar que assombra as equipes $\mathrm{SF} /$ NASF. Na construção do Projeto Terapêutico Singular (PTS) procurou-se parceria com instituição religiosa frequentada pela família, CRAS/CREAS e CAPS Adulto. O processo de produzir uma série de cuidados para este paciente foi longo, sofrido, envolveu muitas conversas, acordos, mudança de compreensão familiar no que tange à pessoa deficiente. No momento, ele vive com mais dignidade dentro de seu grupo familiar.

De modo geral, para atender à complexidade das situações presentes nesses casos, a estratégia encontrada foi ampliar a micro-rede (entendida como rede da Atenção Básica e família) para uma macro-rede territorial ampliando o suporte para as intervenções que se fizeram necessárias. Nesse processo, diferentes questões permeiam a formação de redes de cuidado, relativas aos seguintes aspectos:

\section{a) Diferentes modelos de atenção em saúde}

Uma das dimensões que atravessam a formação de redes de cuidado diz respeito às noções de saúde que fundamentam a prática dos diferentes atores que compõem o processo de trabalho, muitas vezes antagônicas e conflituosas. A partir da década de 1980, a OMS incorpora a concepção de promoção de saúde, que não restringe a noção de saúde à ausência de doença, buscando a atuação sobre as condições de vida da população, em ações necessariamente intersetoriais. Desde 2006, o Ministério da Saúde incorpora esse princípio, na Política Nacional de Promoção de Saúde ${ }^{6}$, fortalecendo os princípios do SUS que já previam uma concepção de saúde, que abrangesse as condições sociais, econômicas, políticas, culturais, enfim, as múltiplas dimensões da vida.

A experiência de atuação de fazer saúde no território demonstra que não é possível ignorar que a saúde está envolvida com vários setores da vida, implicando desta forma uma articulação com outros serviços. Dessa forma, a interlocução do SUS com outras políticas sociais é imprescindível quando se atua numa perspectiva de prevenção e promoção de saúde, e não apenas de tratamento à doença.

Apesar das mudanças ocorridas nas últimas décadas, que reorientaram as políticas de saúde desde uma visão integral do sujeito, ainda são presentes concepções medicocentradas e que tomam o indivíduo restrito à sua dimensão biológica.

A complexidade da realidade dos casos atendidos não se encaixa ao modelo reducionista médico-centrado, sendo quase que uma obrigação a ampliação do olhar sobre o processo saúde-doença. Os avanços conquistados no campo das diversas áreas acadêmicas, o progresso tecnológico, promoveu, ao mesmo tempo, uma especificidade cada vez maior, mas também requer a aproximação e associação das diferentes áreas de conhecimento. O caminho entre a atuação centrada numa única área de saber e a almejada intersetorialidade real, necessita transpor o campo da unicidade para a multiplicidade.

Para tanto, uma das possibilidades seria não só reconhecer os limites de cada conhecimento, como também abrir-se à influência mútua entre os diversos saberes específicos, e ir além, configurando novas relações, inseridas num dispositivo mais integrado, buscando atenuar quaisquer limites rígidos entre as disciplinas, ao se deparar com tal vulnerabilidade e complexidade.

Nas discussões e reflexões, decorrente da experiência que os casos proporcionaram à equipe, foi possível perceber que diferentes concepções sobre saúde e cuidado convivem entre os diferentes atores da rede, atualizando-se nos processos de formação de redes de cuidado. Dessa forma, apresenta-se o desafio da construção de uma rede de cuidados que supere os modelos tecnicistas e que possa atender as necessidades de saúde de uma pessoa ou população instigada a adentrar a outras dimensões, na busca de um processo de mudança.

\section{b) A questão da resolutividade}

A resolutividade, um dos princípios do SUS, consiste na eficiência e qualidade da oferta em saúde, para uma assistência condizente com as demandas da população, contínua em seus diferentes níveis de complexidade. Assim, a resolutividade busca centrar as avaliações das ações desde as demandas concretas de saúde dos usuários e a capacidade do sistema de saúde oferecer atenção 
adequada, inclusive no que se refere ao acesso ao sistema.

No entanto, quando nos debruçamos sobre casos extremamente complexos, a resolutividade, como um parâmetro do sucesso das ações em saúde, abre diversas questões: como superar a ação focada em resultados, a expectativa de uma resposta pronta e imediata ao problema apresentado? Em casos de alta vulnerabilidade será possível ter uma única resposta estabelecida numa relação causa-efeito? Atender estes casos não é o mesmo que realizar um procedimento cirúrgico, extirpando o mal que o assola. Então, como cuidar do adoecimento do sujeito inserido em sua realidade tão complexa?

No primeiro caso, por exemplo, a cronicidade da condição vivida pelo sujeito colocava em xeque quais eram as possibilidades de "resolução" para o caso. Na situação, em que o usuário se encontrava na iminência da morte, estava dada a questão: qual a responsabilidade da rede de saúde diante de uma situação em que suas ações não poderiam evitar a morte iminente do usuário?

Como resposta, os profissionais foram encontrando importantes parâmetros por meio dos quais guiar as ações, fundamentados em promover a qualidade de vida e a dignidade na morte. Assim, durante todo este processo, criou-se um movimento solidário entre os profissionais que passaram a entender a função primordial de acompanhar o sofrido processo vivido pelo usuário e investir na dignidade possível. Assim, a resolubilidade pede por uma elaboração de PTS amplo e estratégico de assistência à saúde adequada às reais necessidades da população.

\section{c) O princípio da co-responsabilidade}

Outra dimensão que se mostrou de extrema importância na formação de redes é o princípio da co-responsabilidade como norteador das ações e como engajamento de cada profissional e serviço na composição da rede de cuidado. Neste modelo abrangente - onde os vários serviços e instituições conversam, acordam, atuam com coresponsabilidade - o compromisso de processar o trabalho realizado deve necessariamente fazer parte das ações em cada caso, promovendo a saúde mental de todos os envolvidos, o enfrentamento das divergências, o limite que o outro (família ou serviço) impõe e a capacidade de cada um em lidar com o inusitado.

No trajeto de processamento das ações com a rede intersetorial de cuidado que se forma, pode-se avaliar a complementariedade, a interdependência e o sinergismo das ações, a disposição de compartilhar objetivos, decisões e responsabilidade, a necessidade de aprimorar as relações interpessoais e valorizar a comunicação entre os envolvidos definindo com clareza os objetivos e as intervenções.

É grande o desafio de definir objetivos pela(s) equipe(s) na formação de rede intersetorial, pois é quase inevitável gerar insatisfações, encontrar posições cristalizadas, rotulando e deteriorando as relações interpessoais e criando mecanismos de culpabilização para poder superar as frustrações dos "supostos fracassos".

A formação de vínculos e a pactuação de compromissos estabelecem uma co-responsabilidade entre os profissionais envolvidos e a comunidade, que possibilitam novas intervenções no território. A co-responsabilidade implica estabelecer Projeto de Saúde Territorial (PST) voltado para cuidar e gerir alívio de sofrimento, melhorar e prolongar a vida, evitar ou reduzir danos, favorecer a construção de autonomia, melhorar as condições de vida e evitando o abandono e o isolamento do sujeito.

Diante desse modo de fazer saúde no território, entende-se que não deve ser ignorada a perspectiva de que a saúde de uma pessoa ou de um território envolve vários setores, implicando desta forma em articulações com outros serviços, que dizem respeito a outros aspectos importantes da vida.

\section{Considerações finais}

O modelo assistencial de saúde centralizado e hierarquizado por níveis de complexidade não corresponde mais à realidade vivida pelos profissionais de saúde da Atenção Básica. A demanda dos problemas apresentados pela população pede por uma gestão de cuidados com uma maior organização das ações. Parece importante buscar mais recursos físicos, tecnológicos e humanos que possam contemplar as questões de vulnerabilidade e complexidade dos processos de adoecimento.

O caminho para compor um sistema de saúde resolutivo e acolhedor não descarta a necessidade de consultas com médicos especialistas, de atendimentos com outros profissionais da saúde, de procedimentos diagnósticos e terapêuticos e de internações hospitalares. Mas, a construção das relações interpessoais e interdisciplinares precisa ser otimizada. 
Da mesma forma, a responsabilidade pelos cuidados não é tarefa para um único setor. A co-responsabilidade envolve não só as equipes de Saúde Família / NASF, o envolvimento das famílias e da comunidade, como também a possibilidade de compartilhar os cuidados com os diversos serviços de saúde e demais setores públicos. Criar as condições para a co-responsabilidade implica que os profissionais envolvidos possam se apropriar de suas atribuições, refletir sobre sua capacidade de resolução de problemas, abrir-se às novas possibilidades de parcerias, compreender e respeitar cada um dos níveis assistenciais.

Cuidar desta população, portanto, implica uma permanente articulação entre diferentes equipes, serviços e setores, ainda mais quando se reconhece que a complexidade das demandas de saúde está intimamente relacionada com as diferentes dimensões do contexto de vida do usuário. É importante, dessa forma, incrementar a interação entre as instituições e os diferentes serviços de saúde, fortalecendo a parceria com outros serviços públicos e a consolidação da intersetorialidade dos cuidados. Tudo isso está sendo construído, ao mesmo tempo que trilhado permanentemente, pelos protagonistas da atenção em saúde.

\section{Referências}

1. SUPERVISÃO DE SAÚDE FÓ BRASILANDIA. PUCSP Pró-Saúde. [Acesso em: 26 de jul. de 2016]. Disponível em: <http:/www.pucsp.br/prosaude/downloads/territorio/ Tecnica_saude.pdf.

2. Brasil. Ministério da Saúde. Secretaria Executiva Programa Agentes Comunitários da Saúde. Brasília Ministério da Saúde, 2001.

3. Rosa WAG, Labate RC. Programa Saúde da Família: a construção de um novo modelo de assistência. Rev Latinoam Enfermagem 2005 novembro-dezembro 13(6):1027-34. Disponível em: http://www.scielo.br/pdf/rlae/v13n6/v13n6a16. pdf. Acesso em: 12 ago. 2016.

4. Brasil. Ministério da Saúde. Secretaria de Atenção à Saúde. Departamento de Atenção Básica. Diretrizes do NASF. Brasília: Ministério da Saúde, 2009. Disponível em: http://bvsms.saude. gov.br/bvs/publicacoes/caderno_atencao_basica_diretrizes nasf.pdf. Acesso em: 18 ago. 2016.

5. SÃO PAULO. Secretaria de Planejamento Regional do Estado de São Paulo. Fundação Sistema Estadual de Análise de Dados. Índice Paulista de Vulnerabilidade Social. São Paulo, 2013. (Disponível em: http://indices-ilp.al.sp.gov.br/view/pdf/ipvs/ metodologia.pdf) Acesso em: 05 jul. 2016.

6. Brasil. Ministério da Saúde. Secretaria de Vigilância em Saúde. Secretaria de Atenção à Saúde. Política Nacional de Promoção da Saúde. Brasília: Ministério da Saúde, 2006. 